\title{
Load Control for Unsteady Gusts with Control Surfaces using the Linear Frequency Domain
}

\author{
Ruben B. Seidler*, Markus Widhalm ${ }^{\dagger}$ and Jochen Wild \\ DLR German Aerospace Center, Braunschweig, D-38108, Germany
}

\begin{abstract}
A method is presented, that is able to mitigate gust loads on an airfoil induced by an incoming gust velocity field. It thereby computes the lift coefficient response to a specified arbitrary gust velocity profile and predicts the required time-accurate control surface deflection. The method uses the linear frequency domain solver to predict frequency responses for the gust and control surface derivative of the lift coefficient efficiently. The frequency responses are computed and then subsequently filled as samples into a surrogate model. For a new flight condition the surrogate model predicts the frequency response by mere interpolation. Because the aerodynamic response on the gust and the behavior of the control surface are known, the aerodynamic lift response and the required flap deflection for alleviation can both be predicted from a given gust velocity field.

The method is thereby able to predict the aerodynamic response and a time-accurate deflection for any flight condition in the design space within milliseconds. Results of the method are shown and analyzed on a 2D profile of a transonic airfoil with an implemented plain flap. The parameter studies were made in low speed with variation of Mach number, Reynolds number, angle of attack, flap chord size and initial flap deflection. In comparison to unsteady Reynoldsaveraged Navier-Stokes computations, the presented method can predict the aerodynamic responses with the same accuracy and it saves more than 6 orders of magnitude in computation time. Using the linear frequency domain solver it is also able to predict the arising unsteady aerodynamic behavior and still cover the viscous effects in the flow.
\end{abstract}

\section{Nomenclature}

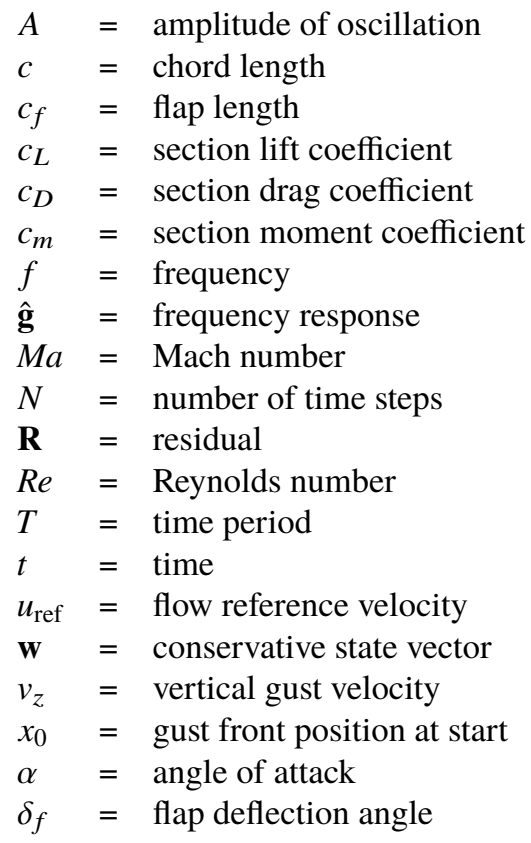

\footnotetext{
* Research Engineer, Institute of Aerodynamics and Flow Technology, Transport Aircraft, ruben.seidler@dlr.de

${ }^{\dagger}$ Research Scientist, Institute of Aerodynamics and Flow Technology, CASE branch, markus.widhalm@dlr.de

${ }^{\ddagger}$ Research Scientist, Institute of Aerodynamics and Flow Technology, Transport Aircraft, jochen.wild@dlr.de, Senior AIAA member
} 


$\begin{array}{ll}\lambda & =\text { gust wavelength } \\ \omega & =\text { angular frequency } \\ \phi & =\text { phase shift }\end{array}$

\section{Introduction}

$\mathbf{T}^{\mathrm{N}}$ modern aviation, new transport aircraft tend to have a higher wing aspect-ratio and a more flexible wing in order Ito increase its aerodynamic efficiency. Thereby the fuel consumption is reduced, which leads to lower emissions and a more efficient aircraft. Due to the high span width of these wings, essential maneuvers and gusts induce additional forces and moments on the aircraft. Especially for the wing bending moment, the aircraft structure needs to be sized accordingly, which is directly related to the weight of the structures in the wing and the overall aircraft. Load alleviation techniques aim to reduce these additional loads and thereby carry a high potential of substantial weight savings for modern aircraft wing structures. This would allow more payload or weight savings due to smaller wings, high-lift systems and control surfaces.

Active load alleviation techniques use control devices of the aircraft, such as ailerons, spoilers and elevators, to actively affect the flow on the aircraft and decrease structural loads. In this work a load alleviation system is introduced, which uses existing and already integrated high-lift systems and ailerons in order to cover a wide area of the wing span and have a strong impact on the span-wise load distribution. The focus of the system is the load alleviation of gusts, since the aircraft has to respond immediately and the system has to cover unsteady aerodynamic responses. The method must be able to accurately predict the aerodynamic influence of the gust on the aircraft and then be able to influence the loads to the desired state using the local control surfaces.

One condition is that the system needs to react extremely fast, since the time between detection of the gusts by sensors and the gust inducing loads on the aircraft is within the scope of milliseconds. Second, the load alleviation system needs to be viable in the complete flight envelope and therefore has to cover all possible flight conditions of the aircraft. It also has to cover the unsteady aerodynamic effects for the gust and the control surface response in order to accurately predict the aerodynamic behavior of the wing. Unsteady Reynolds-averaged Navier-Stokes (URANS) equations are state-of-the-art flow simulations in the industry and are able to predict these unsteady aerodynamic effects. Nevertheless, they are computationally too expensive and the properties of a computation for one flight condition have no reusability. Therefore, computations done by the so called linear frequency domain (LFD) solver are used in a surrogate model (SM) to achieve severe time savings. With the LFD solver based on one steady RANS solution, the derivatives of a gust or a control surface can be computed for various frequencies, so that the frequency response is known for a defined frequency band. By transformation of a time signal into the frequency domain using a fast Fourier transform (FFT) algorithm, it can be combined with the frequency response for the defined input variable to get the response behavior in the frequency domain. By transformation back into the time domain using an inverse fast Fourier transform (IFFT) the time signal of the response variable, in example the lift coefficient, is received. In order to reuse the computations of the LFD solver, the frequency response are filled as samples in a surrogate model at many different flight conditions in order to cover all aerodynamic parameters of the flight envelope. For a new flight condition, the system then efficiently computes the associated frequency response by mere interpolation. Therefore the system is able to predict the aerodynamic gust behavior and the control surface behavior for arbitrary time signals within milliseconds. It covers unsteady aerodynamic effects and is in consequence able to simulate fast gust responses and flap movements, which are required for the load alleviation of gusts.

A requirement for the method is that the incoming flow velocity field is known, either by definition in the numerical simulation or by measurement of the flow velocities. Regan and Jutte [1] showed that the development of technologies for atmospheric sensors and aircraft responses reached a high level of maturity and that now active load alleviation is becoming a crucial part for the design of new aircraft. Different approaches for the methodology of the control-law system have proven to be successful, but non is yet superior to the others. Wildschek et al. [2] introduced an adaptive feed-forward controller based on robust feedback of modal accelerations. They were able to prove the validity of the method and highlighted the improved performance in comparison to a structural feedback control system. Giesseler et. al. [3] developed a load alleviation system, which required the look ahead measurement of atmospheric disturbances by a light detection and ranging (LIDAR) system and predicted gust and actuation input signals by a simplified linear time invariant model. They stated a great potential of the model itself, however, they put emphasis on the possible problems arising with measurement errors and performance issues. Pusch, Knoblach and Kier [4] optimized the parameters of a feedback controller and the geometry for the aileron in order to increase the effectiveness of the gust load alleviation system and achieved a performance improvement of $9 \%$. They demonstrated that a well functioning load alleviation 
system needs to have a control surface on the outer wing and one on the inner wing so that essential parts of the load distribution of the wing-span can be influenced by the control system. Bagheri, Jones and Gaitonde [5] developed a model to predict gust responses using a reduced model of the LFD solver and could show that, for a series of 1-cosine gusts, their model proved a good agreement with the original system. Comparisons between LFD results and Double Lattice Methods (DLM) for gust encounter simulations on the NASA Common Research Model (CRM) were done by Kaiser, Friedewald and Nitzsche [6] and showed good results in terms of time savings and accuracy for applications in the linear gust regions. Kaiser et al. [7] also demonstrated that strong nonlinear effects occur on the wing for high gust amplitudes and that the strongest wing bending moments were not found for the greatest gust lengths, as predicted by their time-linearized approach. Bekemeyer, Thormann and Timme [8][9] used the LFD method to compute the gust responses on a 3D geometry of a large civil aircraft and achieved two orders of magnitude of time savings in comparison to time domain methods. Bekemeyer et. al. [10] also worked out a reduced-order model, which could simulate gusts through hyper-reduction of unsteady computation data for a wide range of flow condition. In comparison to LFD methods it was therefore able to simulate also dynamic non-linearities in the flow.

In order to use the control surfaces effectively, the aerodynamic behavior for the control surface deflections needs to be predicted in a very short time and with a high accuracy in comparison to state-of-the-art URANS computations. Thereby many different approaches for the simulation technique of control surfaces were developed in the past. Ghoreyshi and Cummings [11] used the reduced order model to simulate the unsteady flow field of moving control surfaces and showed that unsteady effects influence the amplitude and phase lag of the aerodynamic loads for moving control surfaces significantly. The surrogate model used in this work was introduced by Seidler et al. [12], which is able to predict unsteady aerodynamic responses for arbitrary and fast control surface deflections within milliseconds.

In this paper the surrogate model of the aerodynamic gust and control surface behavior are now applied for incoming arbitrary gusts in order to fast and accurately mitigate the effect of the gust on the wing. It is explained how the gust response of the wing is predicted in the frequency domain using the advantages of the LFD solver. It is then shown how both surrogate models, for gust and for the control surface, are implemented in the overall process to achieve the ideal flow control. For validation of the model, results of the LFD process are compared to URANS computations on a 2D outboard section of a transonic wing. Numerical results are computed in the subsonic flow region and representative for control surfaces a plain flap is shown for different flight conditions and flap sizes. To deepen the understanding of the model, the possibilities and the limitations are worked out and analyzed in detail.

\section{Gust Simulation in the Frequency Domain}

First the surrogate model is presented, which is able to accurately predict the airfoil response for an incoming arbitrary gust. It thereby decomposes the velocity profile of the gust into its frequency components and computes the lift coefficient response on the airfoil for a given flight condition.

\section{A. Definition of the Gust}

A gust is an atmospheric velocity perturbation and is modeled as a disturbance velocity field. In this work the gust has only vertical velocity components relative to the wing profile. In Fig. 1 an exemplary gust description near the airfoil is shown. On the left side the gust velocity profile $v_{z}(x, t)$ is shown, which describes the gust shape in lateral direction. In the simulation with a fixed wing geometry, the gust always moves with the flow stream velocity $u_{\text {ref }}$ in the downstream flow direction, so that a realistic simulation of a movement of the wing through the gust is achieved. Furthermore, the gust profile always starts at $x_{0}$, which is here in front of the nose of the profile in order to get a gust-free flow at the beginning of the simulation at $t=0 \mathrm{~s}$. In the load control system the shape of the gust velocity profile is known before the gust touches the airfoil, either by definition of gust load cases in the simulation or by a look-ahead measurement in reality. Thereby, a vertical gust velocity time signal $v_{z}\left(x_{0}, t\right)$ is determined, which describes the amount of the vertical gust component on a fixed location at the leading edge $x_{0}$ of the airfoil. On the right side a model of a plain flap is shown, where the downward deflection of the control surface is defined as a positive deflection angle $\delta_{f}$. Since the presented surrogate model is working in the frequency domain, the input time signal of the gust velocity profile $v_{z}(t)$ is now converted into the frequency domain. A FFT algorithm from Cooley and Turkey [13] is used, in order to divide the time signal into its frequency components $\hat{v}_{z}\left(i \omega_{j}\right)$ :

$$
\hat{v}_{z}\left(i \omega_{j}\right)=\sum_{n=0}^{N-1} v_{z}\left(t_{n}\right) e^{-i \omega_{j} t_{n}}, \quad t_{n}=n \Delta t, \quad \omega_{j}=j \frac{2 \pi}{N \Delta t}, \quad j=0,1, \ldots, N-1
$$




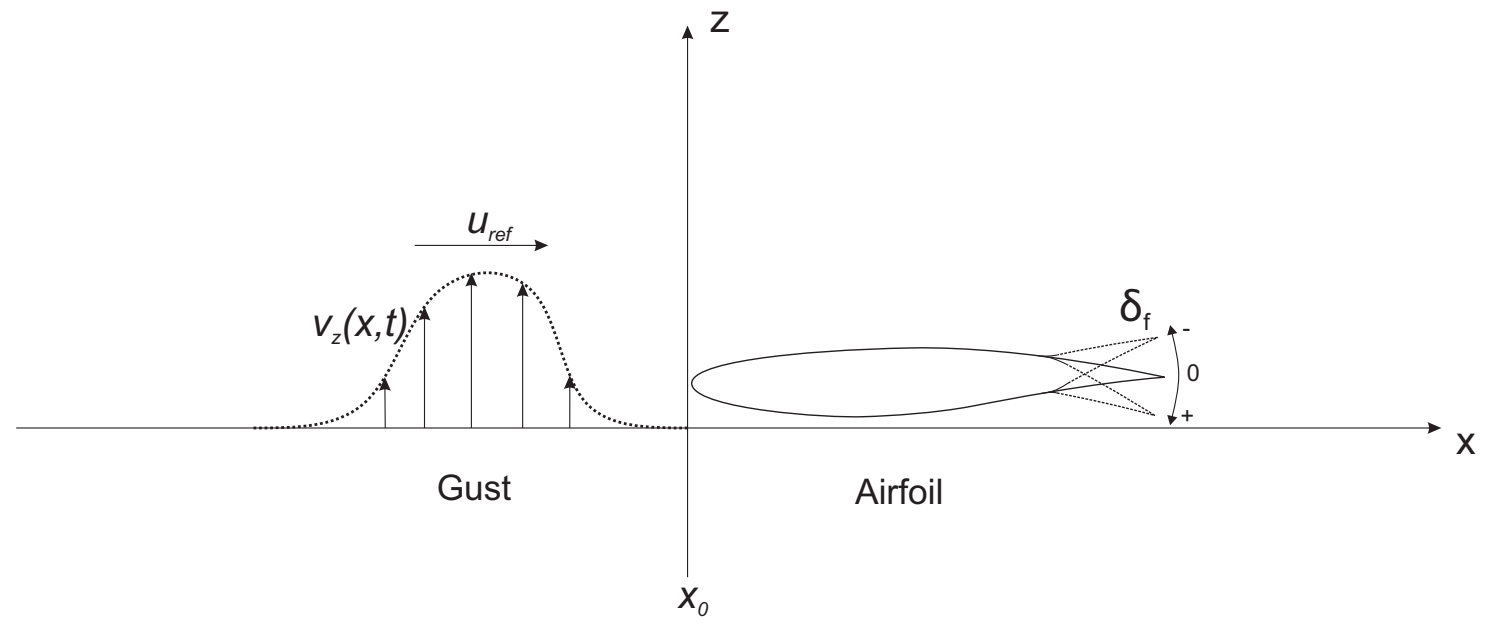

Fig. 1 Sketch of the movement for the vertical gust over the 2D airfoil.

Here, $N$ is the number of time steps, $t_{n}$ are the time steps of the time signal and $T$ is its period length. Smaller time step sizes $\Delta t$ hereby lead to a higher maximum frequency and a higher time period length $T$ leads to a smaller frequency step size $\omega_{j}-\omega_{j-1}=\frac{2 \pi}{T} . \omega$ is the angular frequency for every sinusoidal component in the frequency domain. The gust shape for a sinusoidal gust with a fixed frequency is described as:

$$
v_{z}(x, t)=A\left(v_{z}\right) \sin (\omega \cdot t+\phi(x))
$$

The amplitude $A\left(v_{z}\right)$ and the phase shift $\phi$ define the sinusoidal oscillation for every frequency. Since the gust moves with the speed of the flow stream velocity $u_{\text {ref }}$, the wavelength for a sinusoidal gust can be determined with:

$$
\lambda=\frac{u_{\mathrm{ref}}}{f}=\frac{2 \pi u_{\mathrm{ref}}}{\omega}
$$

In this equation $f$ is the frequency and $\omega$ is the angular frequency. In order to show the decomposition from time domain into frequency domain, a model for the fast Fourier transform results are shown in Fig. 2. In the figure an arbitrary time signal of a vertical gust profile is depicted, which is then shifted into the frequency domain by the FFT. For every frequency component for $f=0 / T, 1 / T, 2 / T, \ldots$ the respective amplitude $A\left(v_{z}\right)$ and the phase shift $\phi$ are shown on the right side. As an example the sinusoidal profile of the $f=2 / T=0.4 \mathrm{~Hz}$ component is shown with its amplitude of $0.031 \mathrm{~m} / \mathrm{s}$ and the phase shift of $70^{\circ}$. Since the original time signal has a length of $T=5 \mathrm{~s}$, for a frequency of $0.4 \mathrm{~Hz}$ two full periods of the sine are achieved within the time.

The spectra represent the decomposition of the original signal into the frequency domain and therefore the phase and the amplitude for different frequencies from the FFT vary heavily and cover up wide ranges of phase shifts and amplitudes. However, for example the different phase shifts only originates from the mathematical decomposition and have no further physical information on the gust. The value for a frequency of an FFT at $0 \mathrm{~Hz}$ is special, because it describes the arithmetic mean and therefore the average value of the time signal. The dataset of amplitude and phase shift for every discrete frequency in a frequency spectrum is used in the further process as an input for the computation of the aerodynamic response in the frequency domain. 

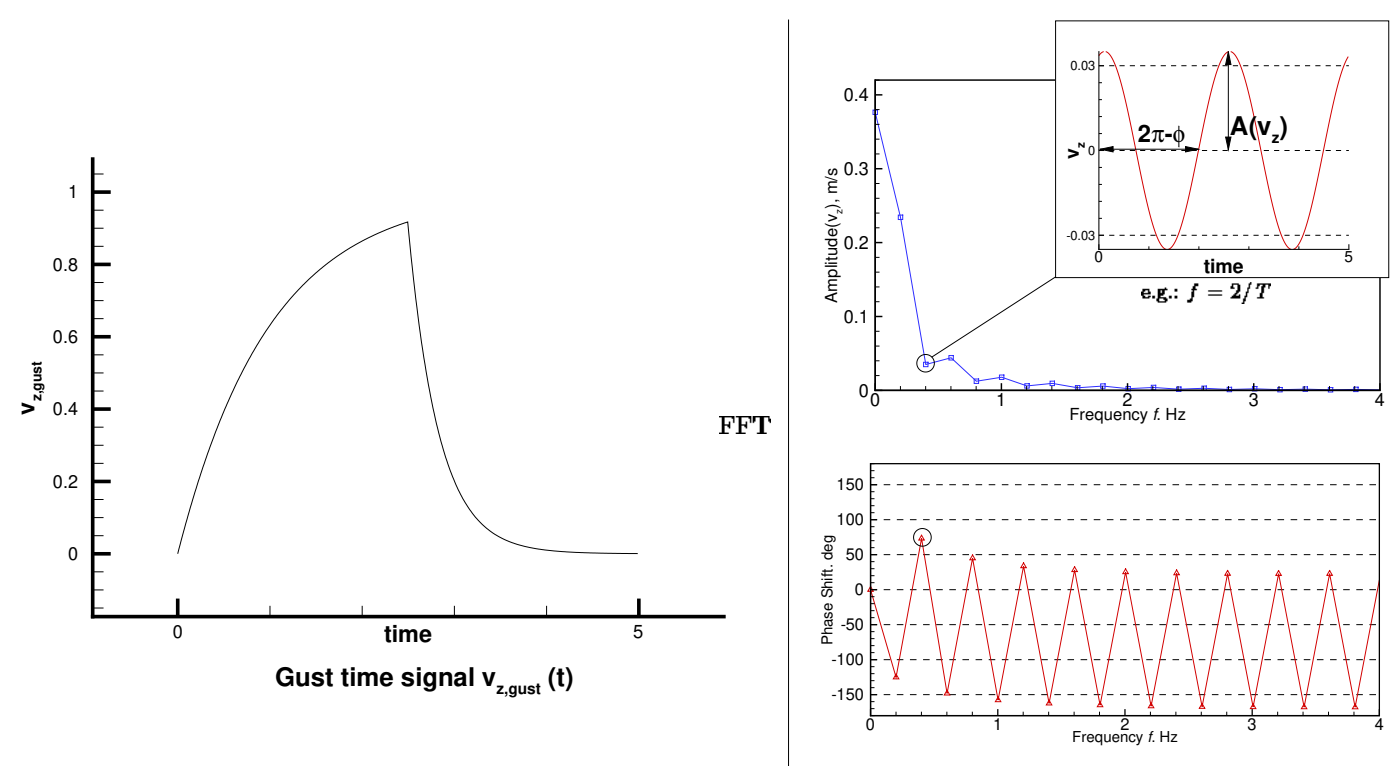

Fig. 2 Arbitrary time domain profile of a gust divided into its frequency components by the fast Fourier transform.

\section{B. Gust Simulation using the LFD Solver}

The gust velocity field is now known in the frequency domain, but the responsive behavior of the airfoil to every sinusoidal gust excitation is unknown. Therefore, the DLR LFD solver is used to predict the dynamic response behavior of the airfoil, which is defined as the frequency response $\hat{\mathbf{g}}$. The frequency response $\hat{\mathbf{g}}$ thereby is a vector, which describes the magnitude and phase shift of the lift coefficient derivative $\partial c_{L} / \partial v_{z}$ for every frequency. The LFD solver is hereby able to efficiently predict these lift coefficient derivatives on the airfoil for gusts with varying wave lengths.

The LFD solver is provided by the DLR TAU code [14] and calculates the first harmonic response of a damped harmonic oscillator. The RANS equations are modeled with a small disturbance approach and by applying the solution of the oscillator in the frequency domain, a complex-valued linear system of equations is obtained. Thus, for a non-harmonic signal (see Fig. 2), that is decomposed into its harmonic components using an FFT, a LFD simulation must be executed for each contributing frequency. The LFD Solver needs one well-converged steady RANS solution for all frequencies. In addition, the control surface deflection, which was treated and shown in Seidler et al. [12], and the disturbance caused by the gust are computed separately and both solutions are then superposed. Here, the gust LFD method is shown in detail. The disturbance caused by the gust can be modeled with the semi-discrete time-dependent flow equations as follows:

$$
\frac{\mathrm{d} \mathbf{w}(t)}{\mathrm{d} t}+\mathbf{R}=0, \quad \mathbf{R}:=\mathbf{R}\left(\mathbf{w}(t), \mathbf{v}_{z}(t)\right)
$$

where $\mathbf{w}$ is the conservative state-space vector of unknowns and $\mathbf{R}$ is the residual of $\mathbf{w}$ and the external disturbance velocities $\mathbf{v}_{z}$ on the mesh induced by the gust. Hereby, the conservative state-space vector $\mathbf{w}$ contains the variables for the flow equations, specifically the mass, momentum, energy and the turbulent viscosity as used by the Spalart-Allmaras turbulence model [15]. By substitution of an unsteady periodic disturbance with a steady state and a small perturbation, the time-dependent variable $\mathbf{w}$ can be described as:

$$
\mathbf{w}(t)=\overline{\mathbf{w}}+\tilde{\mathbf{w}}(t), \quad\|\tilde{\mathbf{w}}\|<<\|\overline{\mathbf{w}}\|
$$

In this equation the vector $\overline{\mathbf{w}}$ describes the steady-state time-invariant part, whereas $\tilde{\mathbf{w}}$ describes the time-dependent perturbation. Since we assume small periodic disturbances, the perturbation has to be distinctly smaller than the 
steady-state part. The residual in Eq. 4 can be expressed by using a first order Taylor expansion:

$$
\mathbf{R} \approx \overline{\mathbf{R}}+\frac{\partial \mathbf{R}}{\partial \mathbf{w}} \tilde{\mathbf{w}}(t)+\frac{\partial \mathbf{R}}{\partial \mathbf{v}_{z}} \tilde{\mathbf{v}}_{z}(t) \quad \overline{\mathbf{R}}:=\mathbf{R}\left(\overline{\mathbf{w}}, \overline{\mathbf{v}}_{z}\right) \approx 0
$$

For a well converged RANS solution the mean residual $\overline{\mathbf{R}}$ is zero in this equation and equivalent to the nonlinear flow solution that describes the aerodynamic nonlinearities [8]. Through substitution of the residual and the vector in Eq. 4 . the equation becomes:

$$
\left(i \omega_{j} I+\frac{\partial \mathbf{R}}{\partial \mathbf{w}}\right) \hat{\mathbf{w}}_{j}=-\left(\frac{\partial \mathbf{R}}{\partial \mathbf{v}_{z}}\right) \hat{\mathbf{v}}_{z, j}
$$

This equation describes the response of the flow variables in vector $\mathbf{w}$ to unsteady periodic gusts, which affect the aerodynamics of the airfoil. The term on the right-hand side $\partial \mathbf{R} / \partial \mathbf{v}_{z}$ is used for the steady-state response and the unsteady response due to variations of the periodic gust frequency $\omega$. It can be seen that for every frequency $\omega$ the LFD solver assumes a linear response behavior of the aerodynamic variables in $\hat{\mathbf{w}}$ to the incoming gust velocity $\hat{\mathbf{v}}$.

For analysis of the unsteady aerodynamic effects of the gusts and control surface deflections in the time domain, an important parameter is introduced, the reduced frequency $k$ :

$$
k=\frac{\omega}{u_{\text {ref }}} \frac{c}{2}
$$

Here, $\omega$ is the circular frequency, $c / 2$ is the semi-chord length and $u_{\text {ref }}$ is the reference flow velocity. Naturally, for the gust the circular frequency is derived from the number of sinusoidal gusts, which are encountered at the leading edge at $x_{0}=0$. The reduced frequency is a characteristic parameter for describing the unsteadiness of the case: At $k=0-0.05$ the flow is said to be quasi-steady [16], which means that the change in disturbance velocity on the airfoil is so slow, that no unsteady effects occur and the gust can be seen as a constant vertical velocity component in the flow field. For $k>0.2$ strong unsteady aerodynamic effects occur due to the high velocity derivatives and heavily influence the pressure distribution of the airfoil and thereby its response behavior.

In Fig. 3 an exemplary frequency response $\hat{\mathbf{g}}$ for the transmission behavior of the gust velocity $v_{z}$ to the section lift coefficient $c_{L}$ on a transonic airfoil is shown. The frequency range covers reduced frequencies from $0 \mathrm{~Hz}$ up to $40 \mathrm{~Hz}$, so that even sharp gusts with high frequency components can be simulated with the model. The frequency response was computed for low-speed conditions with a Mach number of 0.23 , a Reynolds number of $29 \cdot 10^{6}$ and an angle of attack of $2.86^{\circ}$. In the figure the magnitude and the phase shift for this flight condition are depicted. For steady flow conditions at $0 \mathrm{~Hz}$ a gust with a $1 \mathrm{~m} / \mathrm{s}$ amplitude leads to an increase of the lift coefficient of 0.097 . Also there is no phase shift, since there are no unsteady aerodynamic effects, which can cause any disturbances in the response to the gust. At a reduced frequency of 0.01 , unsteady effects arise in the flow and the gust influence decreases up to a lift coefficient gain of $0.02 /(1 \mathrm{~m} / \mathrm{s})$ at $k=1$. There is a delay in the lift coefficient response up to $k=1.5$, in which the phase shift reaches a minimum value of $-80^{\circ}$. This shows that in this flight condition the influence of the gust on the lift decreases and the lag in the response is increasing for higher frequencies. It can be seen that the unsteady effects in the flow heavily influence the aerodynamic behavior of an airfoil in response to incoming gusts.

At this step in the process the frequency components of the incoming gust signal and the transfer function for every gust frequency is known, so that the response behavior of the airfoil can be computed in the frequency domain. The response behavior is computed for every frequency $\omega$ with the equation:

$$
\hat{c}_{L}(i \omega)=\hat{\mathbf{g}}_{\mathrm{gust}}(i \omega) \cdot \hat{v}_{z}(i \omega)
$$

Now the lift coefficient in the frequency domain $\hat{c}_{L}$ is transferred into the time domain by using the inverse fast Fourier transform:

$$
c_{L}\left(t_{n}\right)=\frac{1}{N} \sum_{j=0}^{N-1} \hat{c}_{L}\left(i \omega_{j}\right) e^{i \omega_{j} t_{n}}, \quad \omega_{j}=j \frac{2 \pi}{N \Delta t}, \quad t_{n}=n \Delta t, \quad n=0,1, \ldots, N-1
$$

The variable $N$ is here the number of time steps $t_{n}$ and $c_{L}$ is the response lift coefficient for every time step of the input signal. This process shows, how the time-accurate aerodynamic behavior of an airfoil in response to an incoming gust is computed using the characteristics of the linear frequency domain. 


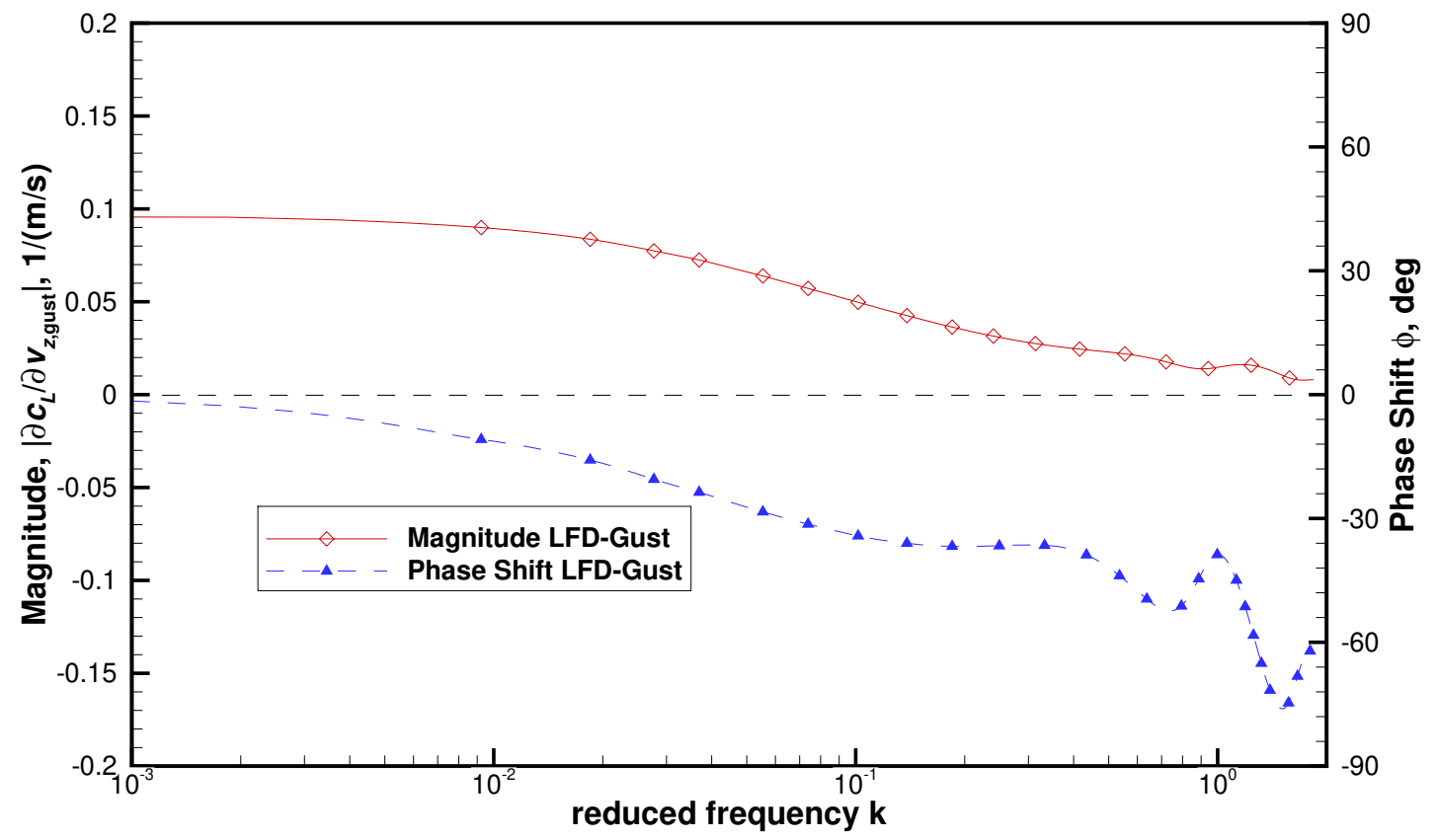

Fig. 3 Frequency response $\hat{g}$ for a gust under low speed flow conditions.

\section{Surrogate Model for Gust Load Control}

In this section the operating principle for the gust surrogate model and the entire control process are demonstrated. It is described how the frequency response data for different flight conditions from the LFD solver are used as a database and then interpolated for new flight conditions in order to save a high amount of computing time. Next, it is shown how the process combines the surrogate models for gust and control surfaces in order to achieve a targeted lift over time for arbitrary incoming gusts by a time-accurate control surface deflection.

\section{A. Surrogate Model for the Gust Response}

The computation for the response behavior needs to be extremely fast and the computation of the frequency response $\hat{\mathbf{g}}$ takes too much time for an in-flight flow control system. Therefore, in this process a number of precomputed frequency responses are implemented as samples in a surrogate model, so that the flight conditions and geometric variances of interest are covered by the parameter space. When a frequency response for a new, yet unknown flight condition needs to be computed, the frequency response $\hat{\mathbf{g}}$ is computed from the sample data by mere interpolation and it therefore only takes time in the scope of milliseconds. The surrogate model can be implemented for different aerodynamic and geometric parameters of interest. The parameters are Mach number $M a$, Reynolds number Re, angle of attack $\alpha$ and an initial flap deflection angle $\delta_{\mathrm{f}}$. The generation of the sampling parameters and the interpolation are done using the DLR SMARTy toolbox. SMARTy thereby stands for Surrogate Modeling for AeRo Data Toolbox and is a software, which provides the handling and fast processing of high fidelity aerodynamic data [17][18]. For this surrogate model, from experience a number of 100 samples was chosen to cover the parameters space for every surrogate model. The sampling data were created using a deterministic Halton sequence [19] to achieve a uniform distribution of sampling data.

In Fig. 4 the samples of the surrogate model for the gust response are shown. In Fig. 4 (a) the steady lift coefficient $c_{L, 0}$ for the 100 different samples are depicted. Due to limitations in the visualization, the parameters Reynolds number $R e$ and the flap chord size are not shown here. It can be seen that within the Mach number limitations of 0.15 to 0.3 and an angle of attack of $-5^{\circ}$ to $10^{\circ}$ the lift coefficient $c_{L, 0}$ varies within a range of 1.8. Naturally, the angle of attack $\alpha$ thereby has a dominant influence on the static lift on the airfoil.

In the second figure (b) the gust frequency responses for 30 of the 100 samples are depicted in a polar plot, where the magnitude and phase shift for every gust frequency are shown. At the frequency of $0 \mathrm{~Hz}$ all samples range between 0.05 and $0.13 c_{L} / v_{z}$ in their magnitude, which defines their quasi-steady behavior for incoming gust with very high wave 


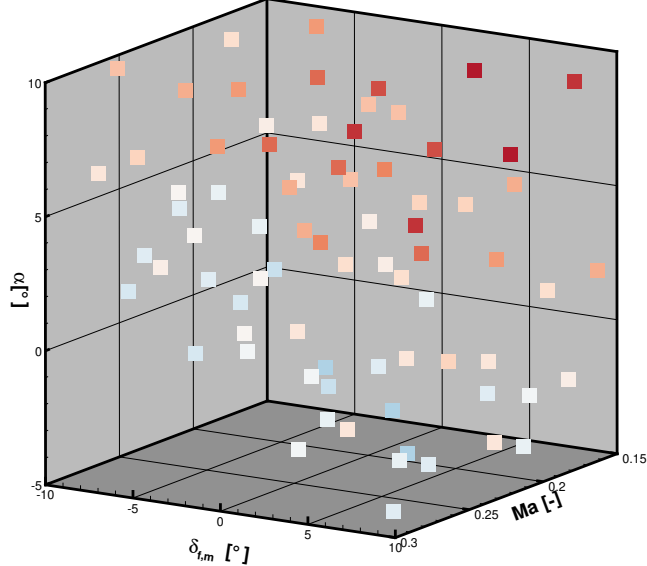

(a) steady lift $c_{L, 0}$

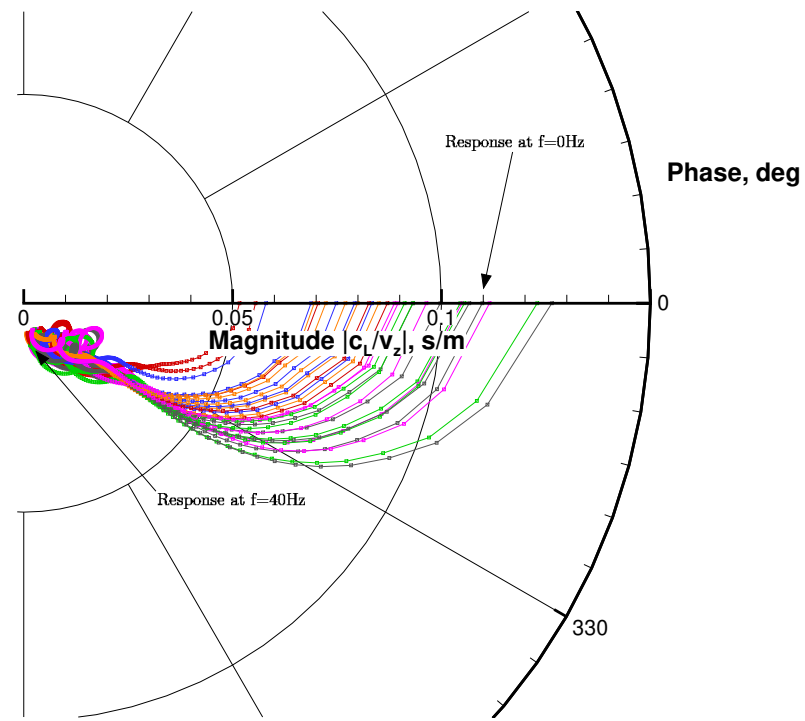

(b) frequency response $\hat{\mathbf{g}}$

Fig. 4 Steady lift $c_{L, 0}$ and frequency responses of the samples used in a surrogate model.

lengths. Also the phase shift starts for all samples at $0^{\circ}$, which means that, as expected, there is no lag between gust and lift response on the airfoil. On the other hand, for high frequencies at $40 \mathrm{~Hz}$ the magnitude decreases heavily up to $c_{L} / v_{z}$, which shows the weaker response to gusts at these wavelengths. Additionally, the phase shift is located between $-15^{\circ}$ and $-90^{\circ}$, which proves that strong unsteady aerodynamic effects generate a lag in the response for these frequencies. Using these samples as data sets, the surrogate models are able to cover a wide range of flight conditions and compute altered flight conditions extremely fast by mere interpolation of the data.

\section{B. Coupling of Gust and Control Surface Surrogates}

Here, the surrogate model is introduced, which describes the dynamic behavior of an airfoil in response to a gust. In preceding works a surrogate model was developed, which could describe the aerodynamic response behavior to a dynamic control surface deflection also using the same assembly of LFD computations and surrogate modeling techniques [12]. Both methods are reversible, so from a lift coefficient time profile $c_{L}(t)$ also a respective gust profile $v_{z}(t)$ or a flap deflection profile $\delta_{f}(t)$ can be computed. Since the frequency response of both, the gust and the control surface, is known for every flight condition, the surrogate model can be used to control the lift response of the airfoil in response to a gust using a countering and immediate flap deflection. The resulting lift response $c_{L \text {,res }}(t)$ of the airfoil is calculated by:

$$
\Delta c_{L, \text { res }}(t)=\Delta c_{L, v_{z}}(t)+\Delta c_{L, \delta_{f}}(t)
$$

In this equation $c_{L, v_{z}}(t)$ is the lift coefficient response originated by the gust at a fixed flap position and $c_{L, \delta_{f}}(t)$ is the lift coefficient response initiated by a dynamic flap deflection without a gust. After the target resulting lift response is preset and the separate gust response is known, by superposition, the equation can be set to compute the required lift coefficient $c_{L, \delta_{f}}(t)$, which needs to be generated by the control surface. By using the frequency response $\hat{\mathbf{g}}_{\delta, f}$ of the flap surrogate model, the flap movement profile $\delta_{f}(t)$ is computed, which is required to control the lift coefficient response for a gust from the actual state to the desired target state. Since both frequency responses are received from the surrogate model, there is no CFD computation needed in the online process and as soon as the gust velocity profile is known, the required flap movement profile can be calculated within milliseconds. In Fig. 5 the framework for the entire feed-forward control system is depicted. The model is split into two parts, on the one hand the offline data generation part, where the surrogate models are filled with samples of frequency responses and on the other hand the fast online time signal computation. In the offline part the sampling parameters and their range limits for the surrogate models are chosen. As stated before we use the Mach number Ma, Reynolds number Re, angle of attack $\alpha$ and the flap deflection angle $\delta_{f}$. The surrogate model for the control surface also uses the flap chord ratio $c_{f} / c$ as a design parameter. These parameters are given to the DLR SMARTy toolbox, where around 100 samples of the frequency response are computed 


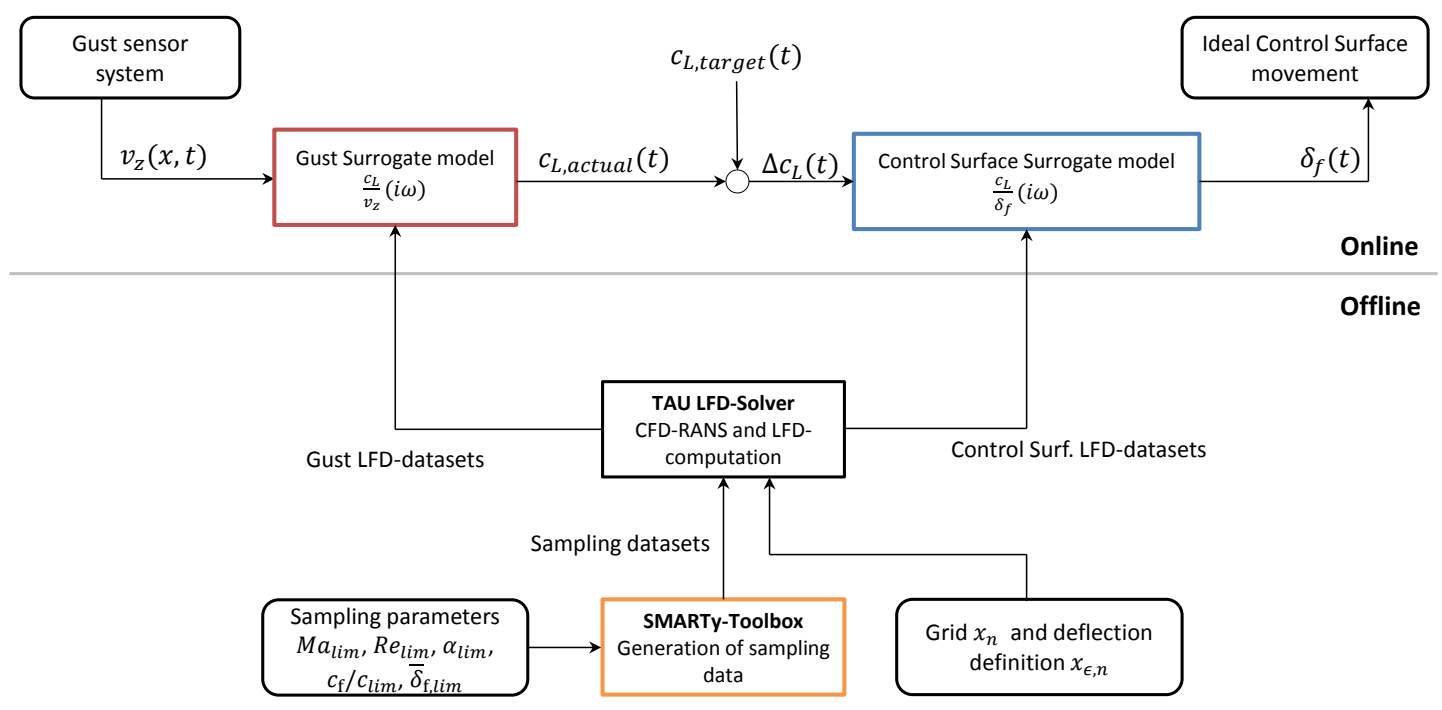

Fig. 5 Framework for the feed-forward flow control system of the control surface for an incoming gust.

to cover up the parameter space. For every one of those samples the TAU LFD solver computes a frequency response, one for the gust and one for the control surface deflection, for 200 frequencies ranging from 0 to $40 \mathrm{~Hz}$. Every frequency response requires a RANS solution at this flow condition, which is computed by the DLR TAU CFD solver [20]. For the control surface frequency response the grid deformation for the flap deflection are given to the LFD solver. For the gust response the grid derivatives are directly calculated and added on the respective grid points by the solver automatically. When the 100 samples for both surrogate models are computed, the sample data are filled into the surrogate model and the offline process is finished (as seen in Fig 4).

In the online process first a gust velocity profile $v_{z}\left(x_{0}, t\right)$ needs to be given as an input and is shifted into the frequency domain using the FFT. For this flight condition the gust frequency response is then interpolated from the surrogate model using a Kriging algorithm. Then the complex multiplication of gust and gust frequency response with equation 9 leads to the resulting actual lift response, which predicts the airfoil load response for the gust-only case. A target lift coefficient response is defined by the user, which describes how the airfoil should respond by using the control surface to achieve the desired loads. The deviation of target and estimated lift coefficient $\Delta c_{L}(t)$ is determined, which quantifies the amount of lift the control surface needs to compensate to achieve the target lift response. The control surface frequency response is provided by the surrogate model and then divided through the frequency components of the deviating lift coefficient in order to get the flap deflection movement in the frequency domain. It is shifted back into the time domain using the IFFT and finally the ideal control surface movement $\delta_{f}(t)$ is predicted to achieve the target lift coefficient response.

\section{Results}

In this section the results for the application of the presented control system are shown. Simulations of different gusts with the LFD surrogate model are demonstrated to show the effects of varying parameter settings for the aerodynamic response of the airfoil. These simulations are compared with URANS computations, which serve as a reference in terms of accuracy. Additionally, LFD-SM and URANS computations are compared in the required computation time in order to demonstrate the advantages of the surrogate model.

\section{A. Validation of LFD-SM with URANS Results}

The results of the surrogate model approach and the unsteady RANS solutions are compared for different testcases. As a realistic geometry, the airfoil of the transonic wing of the Airbus XRF1 aircraft was chosen. The XRF1 is a 
long-range wide-body aircraft, which is provided by Airbus for research purposes. The surrogate model was filled with 100 samples of frequency responses, each covering a gust frequency range of 0 to $40 \mathrm{~Hz}$ with 200 frequencies. The URANS computations were done using 500 to 1000 time steps to cover the unsteady aerodynamic effects for every simulation accurately.

In Table 1 the parameters for the URANS and LFD computations are listed. The following case shows results for the lowspeed surrogate model SM 1 and the computations for case 1 with a Mach number of 0.2 and an angle of attack of $0^{\circ}$. In Fig. 6 the URANS and LFD-SM computations for four different gust profiles are shown. On the lower half the time

Table 1 Parameters for the considered surrogate model and their specific cases.

\begin{tabular}{lcccccc}
\hline Index & Description & Ma $[-]$ & $\operatorname{Re}[-]$ & $\alpha[\mathrm{deg}]$ & $\bar{\delta}_{\mathrm{f}}[\mathrm{deg}]$ & $c_{\mathrm{f}} / c[-]$ \\
\hline SM 1 & plainflap, lowspeed & $0.15-0.3$ & $15-30 \cdot 10^{6}$ & $-5-+10$ & $-10-+10$ & $0.1-0.3$ \\
case 1 & - & 0.2 & $19 \cdot 10^{6}$ & 0.0 & $\bar{\delta}_{f}(t)$ & - \\
case 2 & - & 0.2304 & $21.8 \cdot 10^{6}$ & 2.868 & $\bar{\delta}_{f}(t)$ & - \\
\hline
\end{tabular}

domain of the gust velocity at the airfoil nose $x_{0}$ and on the upper half the resulting lift coefficient response is depicted.

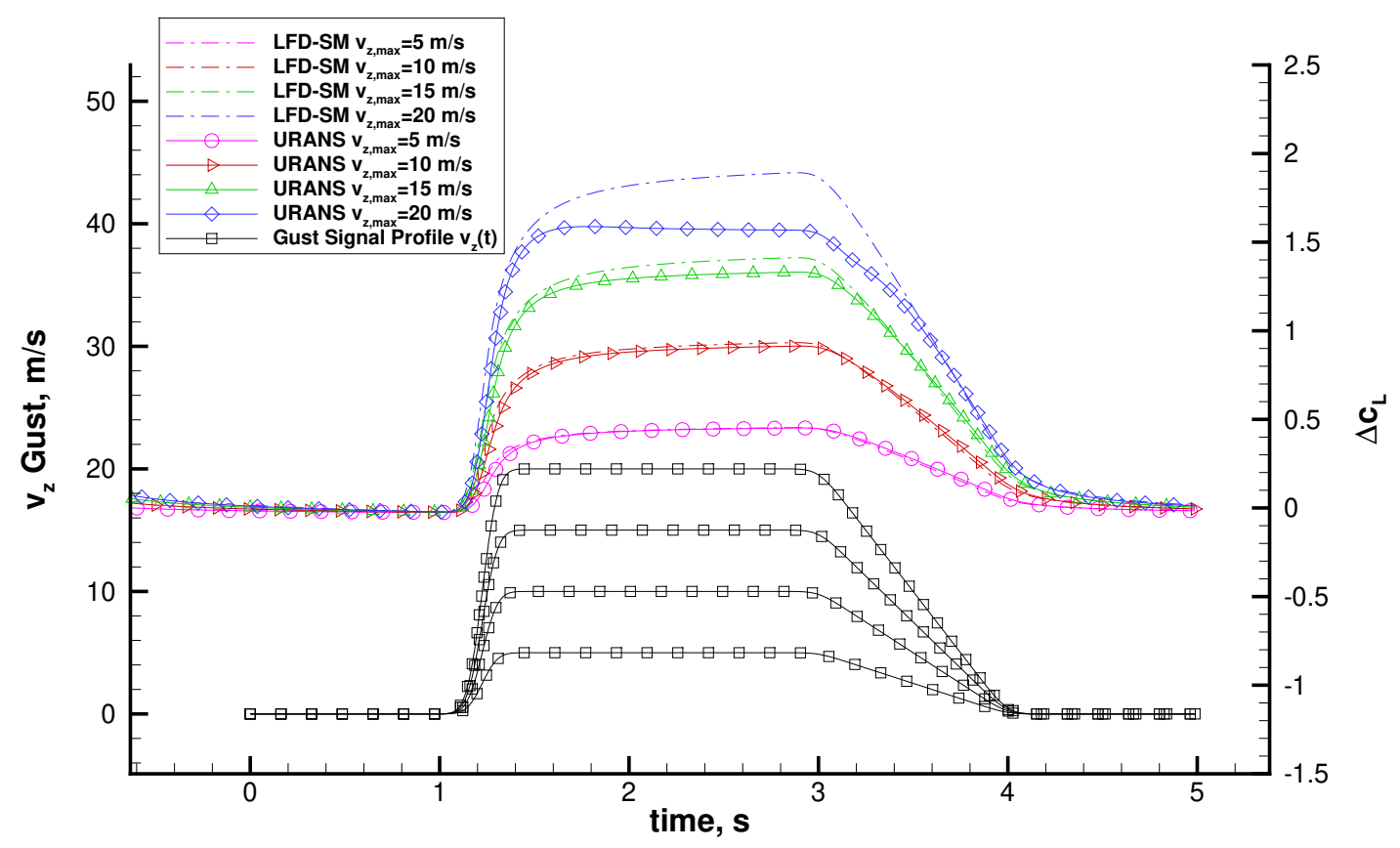

Fig. 6 Variation of maximum vertical gust velocity - comparison between URANS and LFD-SM results.

The computations represent a low speed flight condition on a transonic airfoil. Every gust profile has the same relative velocity $v_{z}$ course, but as a variation the maximum amplitude of the gust is increased from $5 \mathrm{~m} / \mathrm{s}$ to $20 \mathrm{~m} / \mathrm{s}$. The FFT and the LFD always assume the input time signal to be periodic and the response to be without an initial transient phase, as it is always the case for time-accurate unsteady simulations. Therefore, for comparison, the URANS computations are done for 2 to 4 periods of the time signal to reach a swung-in state.

For a maximum amplitude of 5 and $10 \mathrm{~m} / \mathrm{s}$ it can be seen that the lift coefficient response of the surrogate model matches the URANS results with a high accuracy and is able to predict the gust response with the provided LFD data. For a 15 $\mathrm{m} / \mathrm{s}$ maximum amplitude non-linear effects start to occur in the flow and the lift coefficient responses diverge. At $20 \mathrm{~m} / \mathrm{s}$ the flow separates from the airfoil and therefore the lift coefficient response has a strong nonlinear behavior. The LFD model does not have information on the flow separation, since the data were calculated at the steady-state around $\pm 0 \mathrm{~m} / \mathrm{s}$ gust velocity. Here, the LFD method therefore assumes that the flow is still attached to the airfoil and overshoots the predicted lift coefficient response of the airfoil. 
Another specific case for the showing the characteristics of the gust response is shown in Fig. 7 . In this example the gust profile has the same shape for both cases, but they have different period lengths of $T=5 \mathrm{~s}$ and $T=0.5 \mathrm{~s}$. Thereby,

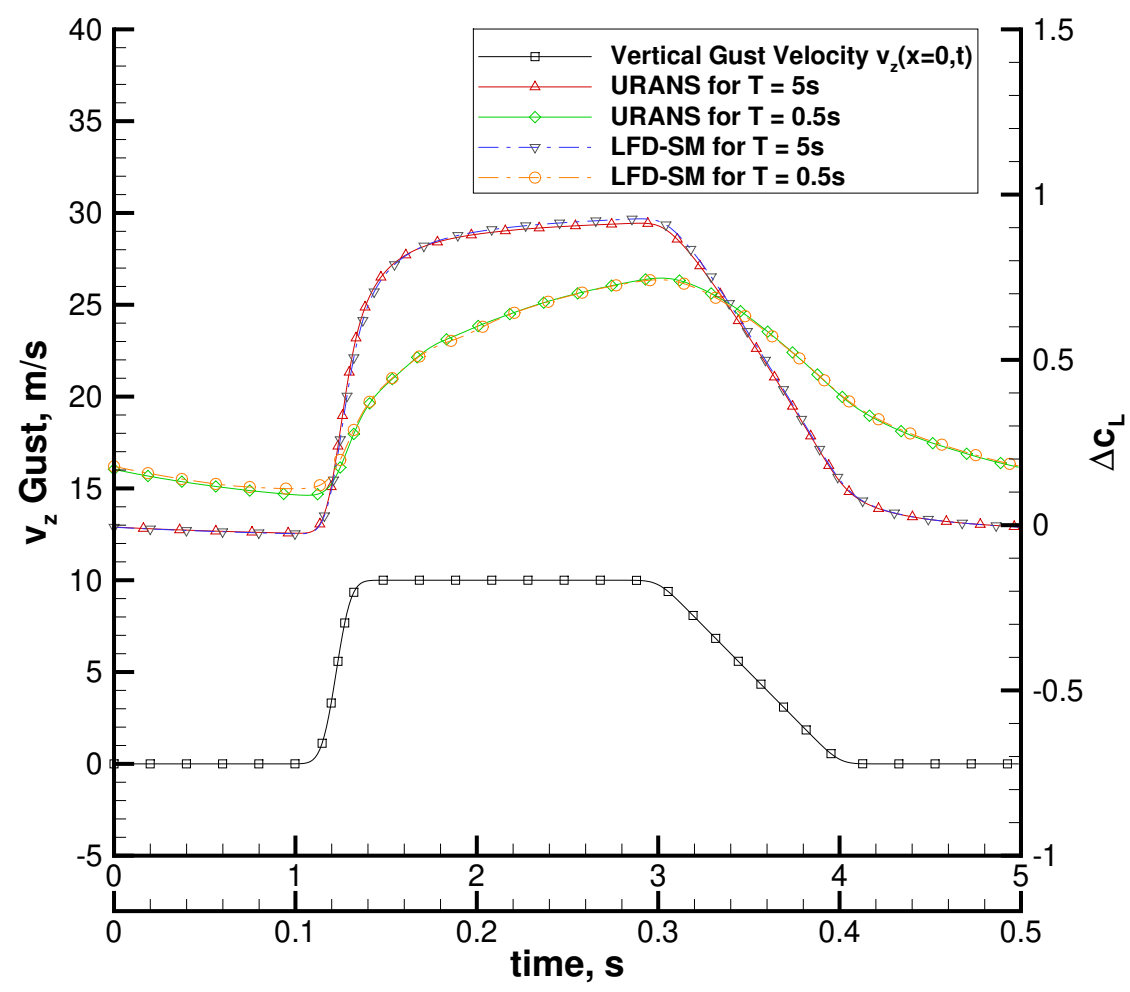

Fig. 7 Variation of period length - comparison between URANS and LFD-SM results.

we can see the effect of reducing the wavelength of a periodic gust, while remaining the shape of the gust profile. The computations were thereby made with a periodic gust profile moving over the airfoil, until the aerodynamics reached a swung-in state in the lift response. It can be seen, that for the quasi-steady case with a gust period length of $T=5 s$, the lift follows the shape of the gust and almost resets to the no gust state after every period length of $5 \mathrm{~s}$. In strong contrast, the gust with a wavelength of $0.5 \mathrm{~s}$ first of all ends with a $\Delta c_{L}$ of 0.2 at every period, because the flow around the airfoil has no time to settle until the next gust arrives. Furthermore, the effect of the gust is smaller, since for these frequency ranges the gust has less influence on the lift magnitude and therefore the lift only reaches a maximum $\Delta c_{L}$ of 0.7 . This examples shows the strong influence of the unsteady aerodynamic effects around the airfoil, which lead to a different behavior for gusts with different wavelengths. However, we can see that the results of the LFD method perfectly match the URANS computation and are able to cover all arising aerodynamic effects in their lift response. This example shows the advantage of the LFD method, which can predict these unsteady aerodynamic effects with high computation time savings.

\section{B. Load Control of the Gust Response using a Plain Flap}

In Fig. 8 an example case of the full flow control system is shown. Similar to before here also a gust with a maximum positive gust velocity of $5 \mathrm{~m} / \mathrm{s}$ is encountered by the airfoil and causes a variation of the lift coefficient over time. The computation were made with the surrogate model SM 1 and the flight condition of the second case (see Table 1 ). The relative lift coefficient response on the gust rises up to $\Delta c_{L}=0.4$ at the gust velocity of $5 \mathrm{~m} / \mathrm{s}$, but within the 2 seconds the flow does not reach a steady-state and the lift is still increasing. The URANS computation for validation matches the response very well, especially in amplitude and behavior. The response of the URANS computation is $0.02 \mathrm{~s}$ behind the LFD model, which is probably due to the implementation of two independent methods and a small deviation at higher frequencies due to the superposition of gust and control surface response.

The desired target state in this case is to minimize the alteration of lift for the airfoil at $\Delta c_{L}(t)=0$, although it is flying through a gust. Using the presented control system, in the figure the required flap deflection $\delta_{f, 1}(t)$ for the alleviation of 


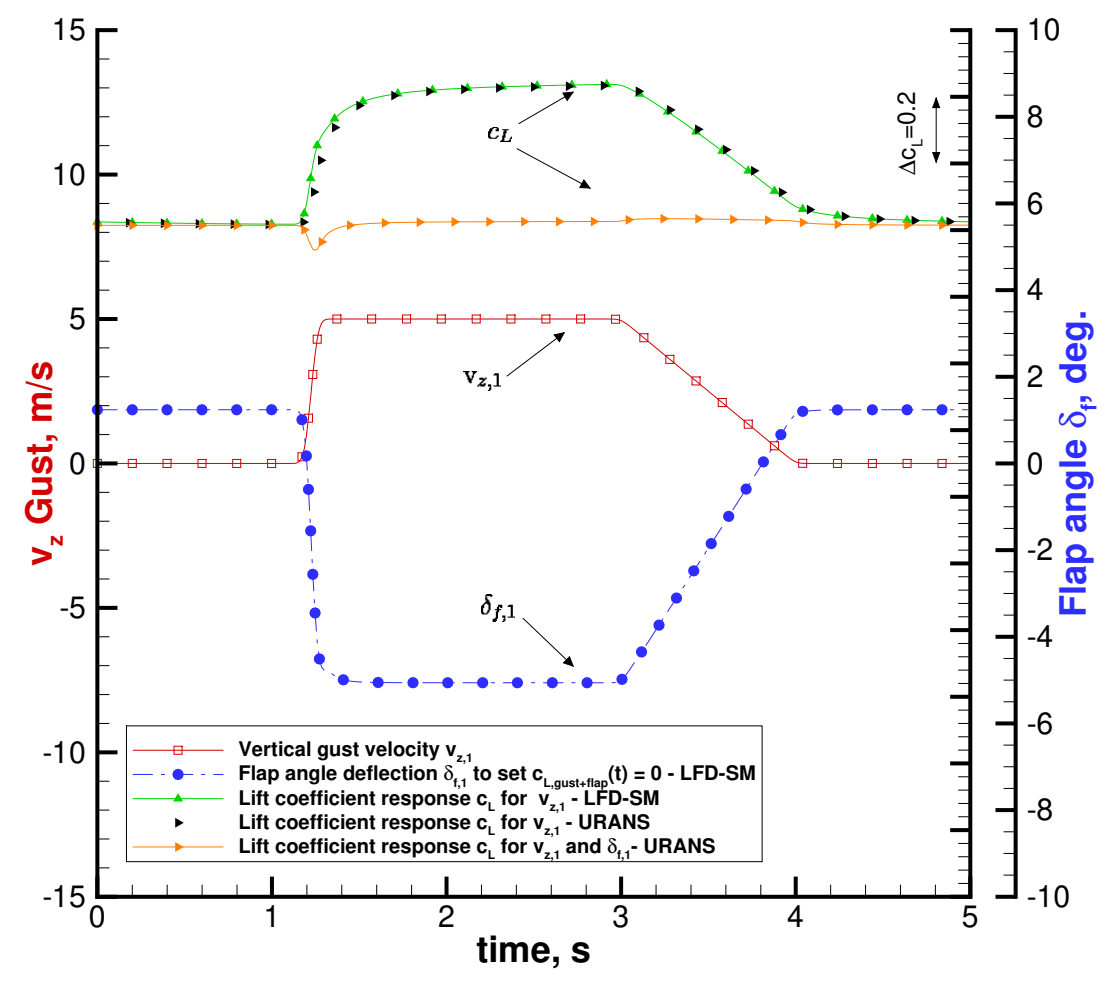

Fig. 8 Example of a gust velocity profile, the respective aerodynamic airfoil lift response and the controlled flap deflection.

the lift is shown. The responsive flap deflection correctly predicts that the plain flap needs to be deflected upwards and decrease the lift to diminish the effect of the gust on the wing. The orange line shows the results of the URANS computation for a simulation with both, the gust velocity $v_{z, 1}(t)$ and the flap angle deflection $\delta_{f, 1}(t)$. At $t=1.2 \mathrm{~s}$ the lift coefficient decreases by 8 lift counts. This occurs due to the prediction of the LFD-SM, which predicts the lift coefficient response for the gust with a time shift of about $10-20 \mathrm{~ms}$ too early and therefore starts to move the flap milliseconds too early. However, in general the orange line for the combined simulation shows excellent results and achieves $\Delta c_{L}(t)=0$ for the most part of the simulation time.

The computation time from $v_{z, 1}(t)$ as an input to $\delta_{f, 1}(t)$ as an output was thereby achieved within 10 milliseconds. In Table 2 the computation times for the offline process and the online process of Fig. 5 are listed. The offline process of computing the 100 samples of the frequency responses has to be done twice, since the surrogate models for flap deflection and gust velocity have to be computed separately. The second online step of computing a flap movement

Table 2 Computational time from the gust velocity field to the flap deflection profile for the LFD-SM.

\begin{tabular}{llc}
\hline Method & Computation steps & Comput. time \\
\hline Offline & 100 samples of $\hat{\mathbf{g}}$ for 200 frequencies with LFD solver $(\approx 2 h)$, parallel & $\approx 100 \cdot 2 h$ \\
Online & $v_{z}(t)$ to $\Delta c_{L}(t):$ FFT, $\hat{\mathbf{g}}_{v z}$ with interpolation $\left(\approx 2 \cdot 10^{-4} s\right)$, Eq. 9 . IFFT & $\approx 1.3 \cdot 10^{-3} s$ \\
& $\Delta c_{L}(t)$ to $\delta_{f}(t):$ iteration(FFT, $\hat{\mathbf{g}}_{\delta f}$ with interpolation, Eq. 9. IFFT) & $\approx 8 \cdot 10^{-3} s$ \\
\hline
\end{tabular}

profile $\delta_{f}(t)$ from a given lift coefficient deviation $\Delta c_{L}(t)$ takes $8 \mathrm{~ms}$, because the frequency response $\hat{\mathbf{g}}$ for the flap derivative is dependent of the mean flap angle and therefore the solution has to be found by iteration. It is important to mention that these time specification only serve as a first indication for the very short computation times of the method. There is a lot more optimization possible in the general computation steps, the application of the surrogate model and the usage of faster programming languages, therefore the method is expected to be even faster in a final case of application.

An additional example is shown in order to point out the side effects of using a control surface to alleviate the lift of 
incoming gusts. Although it reduces the lift and thereby the wing bending moment with the defined control surface deflection, the different influences on the wing pressure distribution of gusts and control surface cannot counterbalance each other at every point of the airfoil. In Fig. 9 the airfoil is affected by one positive 1-cosine gust with a maximum gust velocity of $10 \mathrm{~m} / \mathrm{s}$ for the flight condition of case 1 (see Table 1). The lift induced by the gust $c_{L}(t)$ is alleviated using the control surface deflection $\delta_{f}(t)$, which was computed by the presented LFD surrogate model. In order to

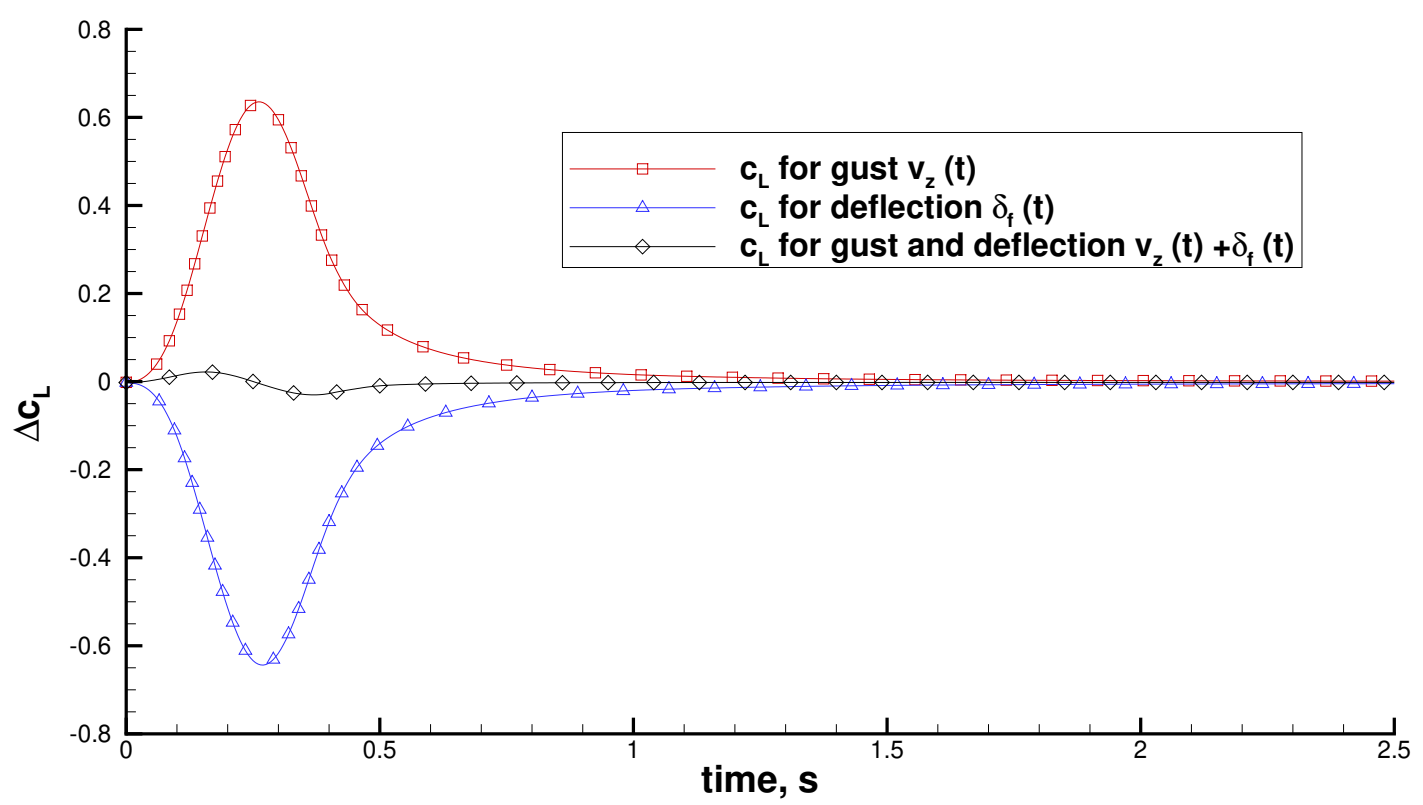

(a) lift coefficient $c_{L}$

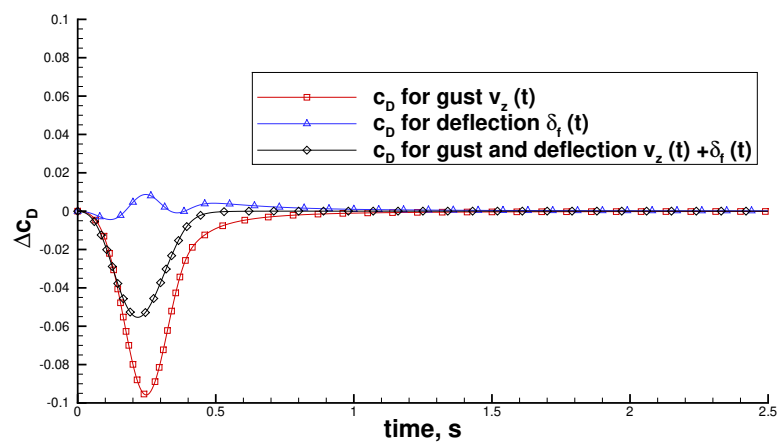

(b) drag coefficient $c_{D}$

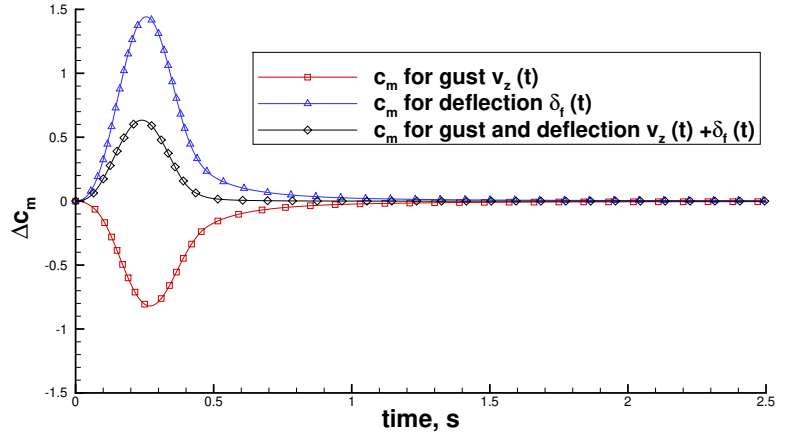

(c) moment coefficient $c_{m}$

Fig. 9 Comparison of lift, drag and moment coefficient for URANS computations of a $1-\cos$ gust with $v_{z, \max }=10 \mathrm{~m} / \mathrm{s}$ for gust and control surface deflection separately and in combination.

single out the different effects, URANS computations were made for only the gust, only the control surface deflection and the combination of both inputs. All three computations are compared in their lift, drag and the moment at $0.25 c$ of the airfoil. Fig. 9 (a) shows the lift response for the three cases: The positive gust leads to an increase in the lift, while the control surface deflection generates an opposing negative lift. As desired, both effects together diminish the lift increase due to the gust, whereas small variations still remain between 0 and $0.5 \mathrm{~s}$. These can be explained in the non-linear behavior of the pressure distribution for high gust velocities and control surface deflections, which cannot be fully covered by the assumption of the linear behavior in the LFD model.

By focusing on reducing the integral lift on the airfoil, two other effects arise on the airfoil: In comparison to the gust only case, in this example alleviating the lift increases the drag (see Fig. 9(b)) and induces a positive moment on the airfoil. The control surface deflection leads to a strong increase of the moment in Fig. 9(c), mostly because of its lever 
arm in relation to the reference point at $0.25 c$. The combination of gust and deflection induces a positive moment around the airfoil. Therefore, when building a load alleviation system, it has to always be taken into consideration, that the pure alleviation of one integral force or moment on the airfoil will always have a strong effect on the other forces on the wing.

\section{Characteristics of the LFD Surrogate Models}

Here, the characteristics of steady and unsteady response of an airfoil to gusts and control surface deflections are shown. Therefore the magnitude and phase of the surface pressure distribution for different frequencies are analyzed. The reduced frequencies of $0,0.1875$ and 0.75 were chosen to analyze the effect of arising unsteady aerodynamic behavior and compare the differences for gusts and control surface deflections. For the gust the magnitude of $c_{p}$ is relative to the vertical gust velocity $v_{z, \text { gust }}$ and for the control surface it is relative to the deflection angle $\delta_{f}$. The computations were made for a Mach number of 0.2 , Reynolds number of $29 \cdot 10^{6}$, an angle of attack of $0.0^{\circ}$ and a set control surface deflection of $1.28^{\circ}$ (see Table 11, case 1 ).

In Fig. 10 the magnitude of the surface pressure distribution for the gust and the control surface deflection are shown. In Fig. 10 (a) it can be seen that the gust has the strongest influence on the front part of the airfoil, whereas the peaks arise at the leading edge near the stagnation point. Additionally, the gust has an extremely small influence on the rear half with $x / c>0.5$ on the airfoil. For gusts with higher frequencies, hence smaller wave lengths, here the influence on the pressure $c_{p}$ is lowering.

The magnitude of the flap derivative in 10 (b) shows a similar trend at the leading edge of the airfoil. The plain flap deflection leads to a strong change of the pressure near the stagnation point and is decreasing for deflections with a higher frequency. However, the control surface deflection also leads to a stronger change in the rear half of the airfoil, where the magnitude reaches a peak of $0.08 c_{p} / \delta_{f}$ for all 3 frequencies. It is important to point out that the unsteady effects of control surface oscillations with higher frequencies flatten out the effect on the leading edge pressure, while the effect on the rear half near the control surface remains the same for the 3 frequencies.

The figures also show, that diminishing the aerodynamic loads of an incoming gust by an ideal control surface deflection will lead to changes in the pitching moment of the airfoil, because their influence on the pressure distribution deviates on different sections of the airfoil.

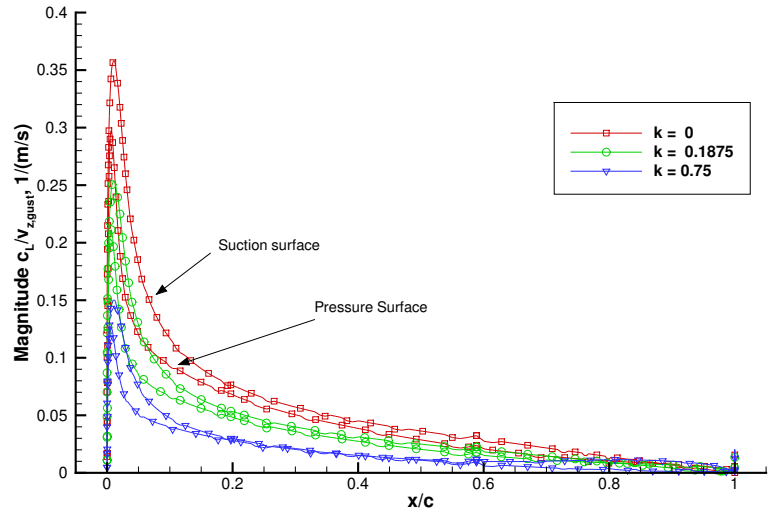

(a) gust derivative

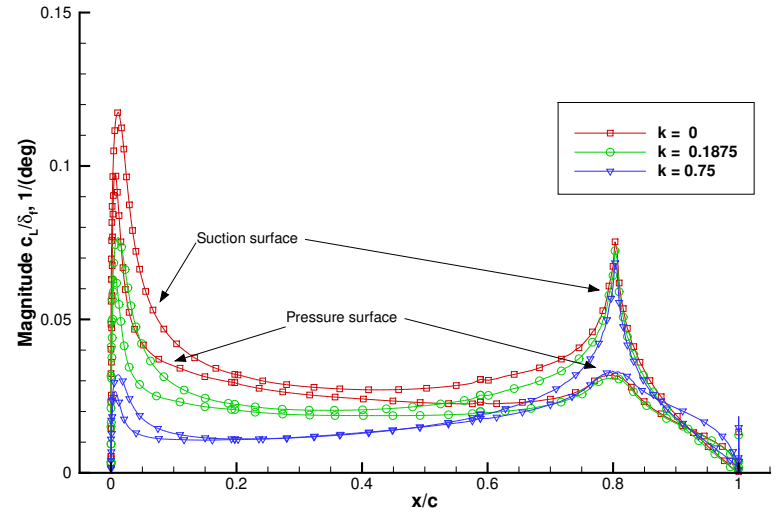

(b) flap derivative

Fig. 10 Surface pressure distribution of the magnitude for varying frequencies.

In Fig. 11 the respective phase shift of the gust and control surface on the airfoil surface are depicted. The phase is shown in degree, which means that a phase shift of $-90^{\circ}$ describes a delay of a quarter period. For gusts in Fig. 11 (a) at $k=0$ the pressure distribution on the suction side of the airfoil has a constant phase shift of $180^{\circ}$, because for an increase in gust velocity, the pressure on the suction side is decreasing. Therefore, in this case input and output have the opposite phase, although there is no actual time shift. On the pressure side for increasing gust frequencies the pressure has a steadily decreasing phase shift. On the suction side for $k=0.75$ the pressure $c_{p}$ has almost a delay of $360^{\circ}$ at the trailing edge of the airfoil, a full period length in comparison to the quasi-steady case. Nevertheless, when looking at the magnitude it can be seen that this section has negligible influence on the overall pressure distribution for the gust.

The Fig. 11 (b) shows the phase shift for the plain flap deflection. Here, the suction side of the airfoil also has 
the opposite phase of $180^{\circ}$ for a reduced frequency of 0 , since the positive downward deflection of the flap leads to a negative increase on the suction surface pressure. In this example for increasing control surface oscillation frequencies, the pressure on the flap section has a preceding response with a phase shift of $45^{\circ}$, whereas a special effect occurs, when moving upstream. The response is delayed up to about $x / c=0.5$ for both sides, whereas for the front section the change in pressure has a lag of $-50^{\circ}$ in response to the control surface movement. In general it can be seen, that in this case for reduced frequencies up to $k=0.75$ the pressure change for an incoming gust is delayed near the leading edge, while some parts of the surface pressure near the control surface are preceding the control surface deflection.

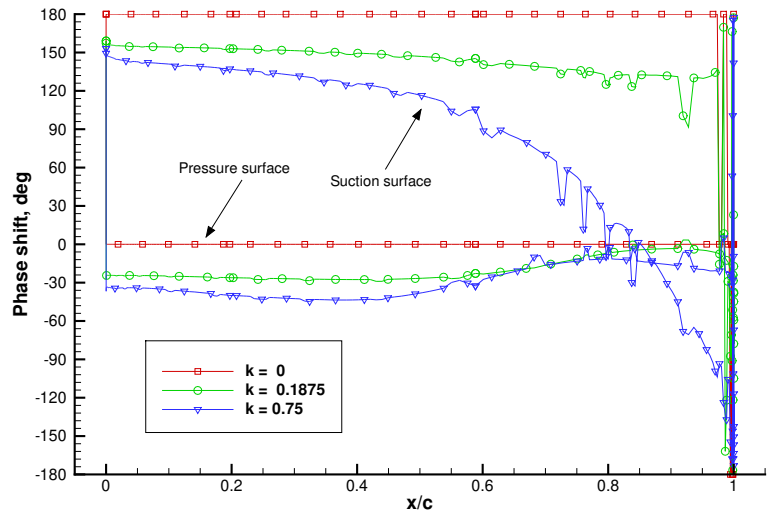

(a) gust derivative

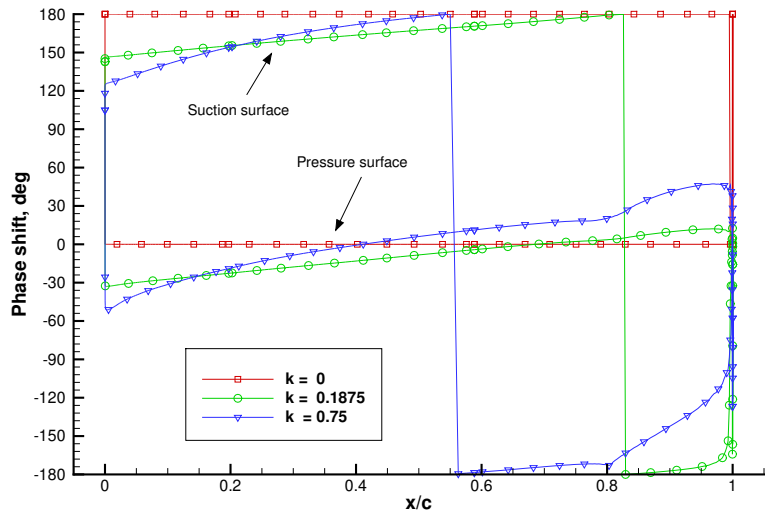

(b) flap derivative

Fig. 11 Surface pressure distribution of the phase for varying frequencies.

\section{Conclusion}

In this article a method is presented, which is able to predict gust and control surface responses fast and with a high precision. It enables the usage for an in-flight load control system, where gust and maneuver loads are predicted and alleviated in a feed-forward system. The usage of the linear frequency domain solver has thereby shown, that it is able to compute the aerodynamic responses for different frequencies efficiently and still cover the viscous and unsteady effects on the airfoil surface. Furthermore, the usage of the frequency responses in a surrogate model allows the wide parameter space for many different flight conditions for the method. The system is able to predict the ideal control surface deflection due to an incoming gust in less that $10 \mathrm{~ms}$, whereas there are still major improvements possible in terms of reducing the computation time.

For analysis, it was shown that the linear frequency domain solver requires a linear response behavior for gust velocities and control surface deflections and starts to deviate for higher gust amplitudes in comparison to URANS computations. Also it was examined that the gust mainly affects the pressure distribution of the leading part of the airfoil near the stagnation point, while the control surface deflection also affects the pressure at the rear part near the control surface. Due to the different effects on the surface pressure distribution of gusts and control surfaces, it must be taken into account that by pure alleviation of the integral lift, the drag is influenced and an additional moment will be induced. In the future, the model will be applied on a realistic use case and validated in wind tunnel tests. Additionally, it will be used for a more complex 3D wing with control surfaces, where the interactions of gusts and extending control surfaces will be analyzed.

\section{References}

[1] Regan, C. D., and Jutte, C. V., "Survey of Applications of Active Flow Technology for Gust Alleviation and New Challenges for Lighter-weight Aircraft," 2013.

[2] Wildschek, A., Maier, R., Hoffmann, F., Jeanneau, M., and Baier, H., "Active Wing Load Alleviation with an Adaptive Feedforward Control Algorithm," AIAA Guidance, Navigation, and Control Conference and Exhibit, 2006. doi:10.2514/6.2006-6054. 
[3] Giesseler, H. G., Kopf, M., Varutti, P., Faulwasser, T., and Findeisen, R., "Model Predictive Control for Gust Load Alleviation," 4th IFAC Nonlinear Model Predictive Control Conference, 2012. doi:10.3182/20120823-5-NL-3013.00049.

[4] Pusch, M., Knoblach, A., and Kier, T., "Integrated Optimization of Ailerons for Active Gust Load Alleviation,” IFASD 2015, 2015.

[5] Bagheri, A. K., Jones, D., and Gaitonde, A., "Linear Frequency Domain Reduced Order Modelling of Aerofoil Gust Response," 46th AIAA Fluid Dynamics Conference, 2016. doi:10.2514/6.2016-4260.

[6] Kaiser, C., Friedewald, D., and Nitzsche, J., "Comparison of Nonlinear CFD with Time-Linearized CFD and CFD-Corrected DLM for Gust Encounter Simulations," International Forum of Aeroelasticity and Structural Dynamics, IFASD $2017,2017$.

[7] Kaiser, C., Quiero, D., and Nitzsche, J., "Quantification of nonlinear effects in gust," International Forum on Aeroelasticity and Structural Dynamics, 2019.

[8] Bekemeyer, P., Thormann, R., and Timme, S., "Linearised Frequency Domain Gust Analysis of Large Civil Aircraft," ECCOMAS2016, 2016. doi:10.7712/100016.2341.5316.

[9] Bekemeyer, P., Thormann, R., and Timme, S., "Frequency-Domain Gust Response Simulation Using Computational Fluid Dynamics," AIAA Journal, Vol. 55, No. 7, 2017, pp. 2174-2185. doi:10.2514/1.J055373.

[10] Bekemeyer, P., Ripepi, M., Heinrich, R., and Görtz, S., "Nonlinear Unsteady Reduced-Order Modeling for Gust-Load Predictions," AIAA Journal, Vol. 57, No. 5, 2019, pp. 1839-1850. doi:10.2514/1.J057804.

[11] Ghoreyshi, M., and Cummings, R. M., "Unsteady Aerodynamic Modeling of Aircraft Control Surfaces by Indicial Response Methods,” AIAA Journal, Vol. 52, No. 12, 2014, pp. 2683-2700. doi:10.2514/1.J052946.

[12] Seidler, R. B., Marten, S., Widhalm, M., and Wild, J., "Efficient Prediction of Aerodynamic Control Surface Responses using the Linear Frequency Domain,” AIAA Journal, 2020. doi:10.2514/1.J058840.

[13] Cooley, J. W., and Tukey, J. W., "An Algorithm for the Machine Calculation of Complex Fourier Series," Mathematics of Computation, Vol. 17, No. 90, 1965, pp. 297-301.

[14] Gerhold, T., "Overview of the Hybrid RANS Code TAU," MEGAFLOW - Numerical Flow Simulation for Aircraft Design, edited by N. Kroll and J. K. Fassbender, Springer Berlin Heidelberg, Berlin, Heidelberg, 2005, pp. 81-92.

[15] Spalart, P., and Allmaras, S., "A One-equation Turbulence Model for Aerodynamic Flows," 30th Aerospace Sciences Meeting and Exhibit, 1992. doi:https://doi.org/10.2514/6.1992-439.

[16] Sears, W. R., "Some Aspects of Non-Stationary Airfoil Theory and Its Practical Application," Journal of the Aeronautical Sciences, Vol. 8, No. 3, 1941, pp. 104-108. doi:https://doi.org/10.2514/8.10655.

[17] Zimmermann, R., and Görtz, S., "Non Linear Reduced Order Models For Steady Aerodynamics," Procedia Computer Science, Vol. 1, No. 1, 2010, pp. 165-174. doi:10.1016/j.procs.2010.04.019.

[18] Franz, T., Zimmermann, R., and Görtz, S., "Interpolation-based Reduced-order Modeling for Steady Transonic Flows via Manifold Learning," International Journal of Computational Fluid Dynamics, Vol. 28, No. 3-4, 2014, pp. $106-121$. doi:10.1080/10618562.2014.918695.

[19] Halton, J. H., “Algorithm 247: Radical-inverse Quasi-random Point Sequence," Commun. ACM, Vol. 7, No. 12, 1964, pp. 701-702. doi:https://doi.org/10.1145/355588.365104.

[20] Gerhold, T., "Overview of the Hybrid RANS Code TAU," Notes on Numerical Fluid Mechanics and Multidisciplinary Design, Vol. 89, 2005, pp. 81-92. 